\title{
An Evaluation of Sunlight-Viewable Displays
}

\author{
Phil N. Day \\ Consumer Experience Displays \& \\ NCR Corp, Dundee. DD2 4SW \\ phil.day@ncr.com
}

\author{
Jim Colville \\ Secure Devices, NCR \\ Financial Solutions Group Ltd, NCR \\ jim.colville@ncr.com
}

\author{
Charlie Rohan \\ Consumer Experience \\ Corp, Dundee. DD2 4SW \\ charlie.rohan@ncr.com
}

\begin{abstract}
There is a need for displays that can be read even in adverse environmental conditions such as under high intensity, directed sunlight. This is particularly important in self-service terminals that may be placed in an exterior location. These terminals are intended to be used by the general public.

This paper reports the results of a study comprising of four evaluations of sunlight viewable displays. These evaluations consisted of 16 and 20 participants, with two additional expert reviews. A comparison is made between two different types of display technology, namely, transflective and high-bright backlit displays, and results are presented of this comparison. Secondary comparisons of different approaches for managing glare and reflections are also presented and discussed. Transflective displays were found to be at least as good as high-bright alternatives and are therefore promoted as a viable technology for daylight-viewable displays to be placed in an exterior environment.
\end{abstract}

\section{Sunlight-viewable transflective display}

\section{INTRODUCTION}

Self-service terminals (SSTs) such as automated teller machines (ATMs) usually have some form of information display incorporated as an integral part of the system. Many ATMs and other SSTs are placed in exterior environments and therefore must be readable under multiple environmental conditions including darkness or full sunlight. The first ATMs had no displays at all, with the next generation having a two-line monochrome display. As ATMs evolved, they moved to using multi-line monochrome, then larger, colour displays.

The modern trend is towards a large colour display, often with a touch element to allow interaction directly with on-screen elements as an alternative to using the screen function keys that were used on earlier generations.

Although standard emissive displays work well in moderate lighting conditions, there are challenges in making them easy to read under bright sunlight. This issue has been well noted in, for example, the design of displays for use in aircraft $[2,6,11]$ and for road vehicles [8] and some efforts have been made to quantify the readability of conventional emissive displays $[3,7]$.

As these displays have increased in size, the issue of glare and reflections on the display has become more of a challenge. Particular problems include glare across a large proportion of the screen, reflections, and hazing. One of the methods to combat this is to add an anti-glare etch onto the glass, or to coat it with an anti-reflective coating. These challenges can still exist when the product is installed in an internal location; for instance a bank branch can often have an ATM towards the front of the branch by a large expanse of glass.

The ability to read a display under bright sunlight is therefore a key requirement for exterior SSTs. It is also known that the performance of backlights used in displays deteriorates with time thus making legibility under bright light even more challenging. An additional factor to be considered is to make the display viewable for all types of installation for all users. This can include multiple installation heights for different ATMs, and also includes drive-up ATMs that provide their own visibility and reach challenges [5].

\subsection{Sunlight-Viewable Display Technologies}

We currently use two main approaches to making a display viewable under bright sunlight.

The first is to have a very bright backlight (a highbright display). In this type of display the backlight (which can emit in the order of $\sim 1700-2000 \mathrm{Cd} / \mathrm{m} 2$ ) is adjusted for the lighting conditions by means of an ambient light sensor, with the intensity of the backlight being reduced significantly in low light conditions (such as at night). However, high bright displays have a number of disadvantages including high power consumption, increased heat generation, increased dimensions, electrical circuit alterations, reduced display lifetime, and deterioration in backlight 
performance - field experience has suggested that after 4 years continuous use, a high bright display only gives approximately $10 \%$ backlight illumination when compared with performance when new.

The second approach is to have a transflective display where a portion of the ambient light is directed back towards the user thus meaning that a less powerful backlight is required [13]. Transflective displays therefore offer the potential benefits of reduced power consumption, running costs and backlight life over high bright solutions; thus resulting in a cost benefit.

These two technologies needed to be evaluated to give some quantifiable comparison of performance between high bright and transflective displays and assess whether there are any significant disadvantages to using transflective displays.

This paper therefore presents a study comprising of four evaluations. These consisted of two evaluations with a number of participants that were conducted to evaluate the differences between the two display technologies, with each evaluation then followed by an expert review of the displays under multiple lighting conditions.

\section{EVALUATION 1: 4 DISPLAYS, 16 PARTICIPANTS}

\subsection{Method}

Four displays were evaluated under the same lighting conditions at the National Physical Laboratory (NPL) in Teddington, UK. The displays were as follows:

A. 12" high-bright concept with anti-reflective (A-R) glass bonded directly onto the display

B. 12" transflective

C. $15^{\prime \prime}$ high-bright

D. 15 " transflective

\subsubsection{Design}

A repeated measures design was used in this study. The principal factor was that of screen type with four levels corresponding to the different screens used. The dependent variables were subjective ratings of the display performance.

Ordering of displays followed a partial counterbalanced approach between participants (Latin square).

\subsubsection{Participants}

16 participants were recruited from NPL employees; 10 male and 6 female with all participants having normal or corrected to normal vision. Age of participants varied from 20-60+ with the majority in the 20-39 age range, and height varied from $15751880 \mathrm{~mm}$ with a mean height of 1734 , and a standard deviation of 95.

\subsubsection{Apparatus and Materials}

All displays were mounted at the same height and angle, which was $1300 \mathrm{~mm}$ from the floor (measured to the top of 12" screen, with the bottom of the screens being aligned) and at $55^{\circ}$ from the horizontal (Figure 1). Displays were labelled by letter only (i.e. participants were not told which technology each display used). The height and angle chosen corresponded to the height and angle of the display in one of our ATMs. All displays were configured to a resolution of $800 \times 600$ pixels, which was the native resolution of the 12" displays. Although the 15 " displays were capable of $1024 \times 768$ pixels, the equipment used to mirror the same test image on all displays meant that all had to be at the lower resolution.

For this evaluation, all four screens had security glass positioned in front of them with an antireflective coating on it. Screens $A, B$ and $D$ had anti-reflective coatings (with screen $A$ having the a-r coating glass bonded directly onto the display thus having no additional air gap). Screen $\mathrm{C}$, however, did not have an anti- reflective coating and instead just had an anti-glare etch on the display, and would therefore be expected to perform worst in terms of reflections.

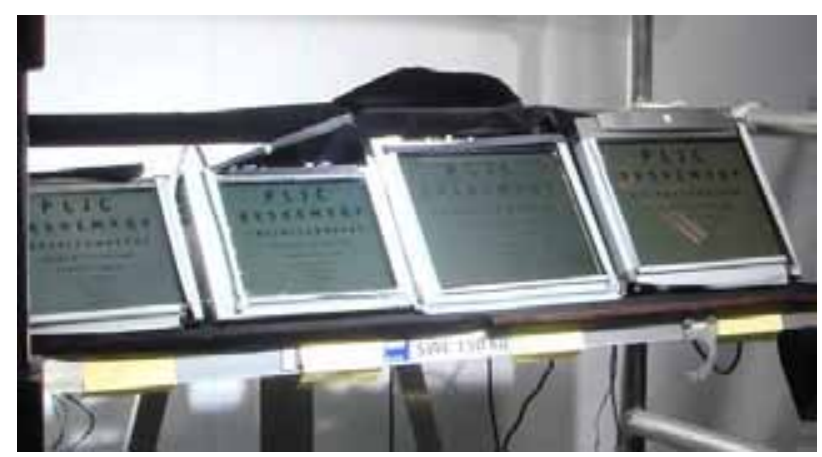

Figure 1: Evaluation 1 Displays

The displays were assessed under a single lighting state due to time constraints (the lamps took a few minutes to cool down after running at bright setting, therefore it was not feasible to change the lighting condition during each test). This lighting state corresponded to bright, midday sunlight and was measured at 100,000 lux, where $80 \mathrm{~K}$ was direct light, 20K was diffuse.

Questions were asked before the evaluation regarding the age, gender, and visual acuity of a participant. After viewing images on each display a participant was then asked to rate them in terms of clarity of image, brightness and comfort. This measure of comfort was explained in terms of how comfortable it would be to view the screen for a longer time (>10 seconds), and was intended to give some measure of the potential discomfort that 
some people can experience particularly with bright, high-contrast displays as noted particularly with test patterns $[12,4]$.

\subsubsection{Procedure}

Each participant completed the pre-experiment questionnaire before viewing four test images (Table 1) and answered a few questions for each display.

Table 1: Evaluation 1 Test Screens

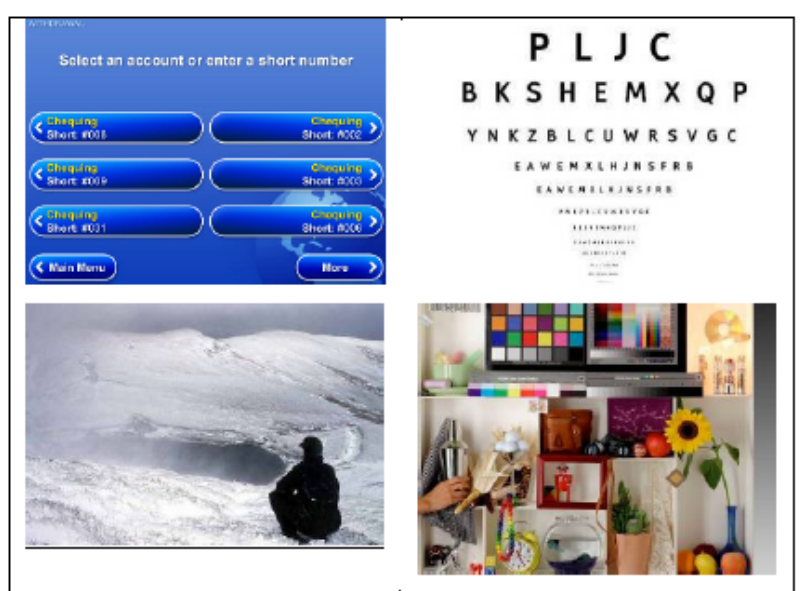

All four test images were displayed on each screen in sequence, with participants being able to step through the images (by clicking a mouse). The test images were selected to evaluate a number of different criteria; namely text legibility on a highcontrast display, colour reproduction, clarity with a complex image, and finally reproduction of a typical screen image for use in an ATM.

Participants positioned themselves $300 \mathrm{~mm}$ from the bottom edge of the display in order to view the images, and were then asked to complete a post-experiment questionnaire eliciting attitudinal measures of perceived performance.

\subsection{Results}

Participants were asked to comment on screen clarity, brightness, comfort and then give a comparison between the displays.

\subsubsection{Screen Clarity}

Participants were asked to rate this on a 5-point scale where $1=$ not at all clear, $5=$ very clear. Ratings are summarized in Table 2, with a ranked score being included to more clearly show the difference between ratings. A higher ranked score is better, with a maximum possible rating of 80 and a lowest of 16 (ranked score $=a^{*} 1+b^{\star} 2+c^{\star} 3+d^{*} 4+e^{\star} 5$ ).
Table 2: Evaluation 1 Screen Clarity Ratings

\begin{tabular}{|l|ccccr|r|}
\hline & $\begin{array}{l}\text { 1 (not } \\
\text { clear) }\end{array}$ & $\begin{array}{l}\text { Rated } \\
\text { as 2 }\end{array}$ & $\begin{array}{l}\text { Rated } \\
\text { as 3 }\end{array}$ & $\begin{array}{l}\text { Rated } \\
\text { as 4 }\end{array}$ & $\begin{array}{l}\text { 5 (very } \\
\text { clear) }\end{array}$ & $\begin{array}{l}\text { Ranked } \\
\text { Score }\end{array}$ \\
\hline A & 0 & 1 & 0 & 5 & 10 & 72 \\
B & 0 & 2 & 4 & 5 & 5 & 61 \\
C & 4 & 8 & 3 & 1 & 0 & 33 \\
D & 0 & 5 & 4 & 7 & 0 & 50 \\
\hline
\end{tabular}

\subsubsection{Screen Brightness}

Participants were asked to rate this on a 5-point scale, but this time the midpoint (3) was the positive result with $1=$ too bright, $3=$ just right, 5=too dim. Again a ranked score is used to clearly show the differences (ranked score $=a^{*} 1+b^{*} 3+c^{*} 5+d^{*} 3+e^{*} 1$ ). Ratings for this and other measures are summarized by means of the ranked scores in Table 3 for brevity.

\subsubsection{Screen Comfort}

Participants were asked to rate this on a similar 5-point scale, where 1=very uncomfortable, 5=very comfortable. Again, a ranked score was used to show differences between ratings (ranked score = $\left.a^{*} 1+b^{*} 2+c^{*} 3+d^{*} 4+e^{*} 5\right)$.

\subsubsection{Comparison Between Screen Ratings}

Ratings for each screen gave a clear indication of preference between the displays. When summarized by means of ranked scores for clarity, brightness and comfort, screen $A$ was rated best, $B$ next, $D$ next and $\mathrm{C}$ was rated worst (Table 3 ).

\section{Table 3: Evaluation 1 Ranked Scores}

\begin{tabular}{|l|rrr|}
\hline Screen & \multicolumn{1}{|l}{ Clarity } & Brightness & \multicolumn{1}{c|}{ Comfort } \\
\hline A & 72 & 57 & 67 \\
B & 61 & 57 & 59 \\
C & 33 & 46 & 32 \\
D & 50 & 47 & 45 \\
\hline
\end{tabular}

\subsubsection{Comparison Between Screen Opinions}

Participants were also asked some general comparative questions at the end of the evaluation. All 16 participants felt that there was a discernible difference between the displays. Fifteen out of sixteen participants stated that screen A (12" highbright with bonded anti-reflective glass) was best, with 1 other participant preferring screen B (12" transflective).

Fifteen participants stated that screen C (15" highbright) was worst, with 1 participant choosing screen B (12" transflective) instead. From comments that participants made, screen C was particularly susceptible to glare problems with $D$ being next worst. This is likely to be due to screen $C$ being the only display that had an anti-glare etch instead of the anti-reflective coating that the other three displays had. 


\subsubsection{Preferred Screen Size}

Participants were asked which size display they would rather use in a self-service terminal such as an ATM. The majority of participants (14 out of 16) said that they would prefer a smaller (12") display, with only one participant preferring the larger (15") display. The remaining participant stated that either size was fine.

Comments from participants suggested that size was not as important as clarity, ability to cope with glare, sufficient colour vibrancy and contrast.

These results were supported by the ratings of clarity, brightness and comfort, and by choices for best and worst screen, all of which suggested that the 12" screens performed better than the 15 " screens.

\subsection{Discussion}

From the ratings of clarity, brightness and comfort display A (which had anti-reflective glass bonded directly onto the display panel) was preferred. However, this was judged to be due to the lack of air-gap in this setup rather than the fact that it was a high- bright backlit display. In fact, the 15" high-bright display evaluated did not have this bonded glass, and was rated less highly by participants. Display B (12" transflective) was the next best in terms of these ratings. Displays $C$ (15" high-bright) and D (15" transflective) were close in terms of ratings, with $\mathrm{D}$ being slightly preferred.

From these results, if the bonded glass display is eliminated, the performance of the transflective displays was at least as good as the high-bright backlit display.

\section{EVALUATION 2: 4 DISPLAYS, EXPERT REVIEW}

\subsection{Method}

The subsequent expert evaluation was conducted under three lighting states; low light (0 lux), moderate light (22 k lux, all diffuse light, equating to an overcast day), and bright (100 k lux, $80 \mathrm{k}$ direct, $20 \mathrm{k}$ diffuse). Viewability was assessed for the range of likely eye heights using worldwide anthropometric models rather than national percentiles as previously presented [10]. This was assessed by the reviewer placing their eyes at the appropriate level for each height, and judging whether all 4 test images were clearly distinguishable. Of particular concern in this assessment was that the extremes of the worldwide population stature (from very tall ambulant consumers down to very small wheelchair-seated consumers) can use the display. Additional assessments were made of text legibility and colour reproduction for these eye heights.

\subsection{Results}

The first stage of this evaluation was conducted under bright light (100 k lux).

\subsubsection{Viewability}

All displays were viewable at all likely user head heights (from 97th 10th percentile Dutch male to percentile UK female in wheelchair); i.e. the display view angles accommodated everyone, with text contrast and colour reproduction being acceptable from all head heights. However, there were lots of glare issues which meant that one had to move around a lot to counteract the effects of glare and to successfully view portions of the display.

Screen A (12" high-bright with bonded anti-reflective glass) was best for glare (i.e. had least glare) followed by $D$ ( 15 " transflective), B (12" transflective), and screen $C$ (15" high- bright) was worst.

\subsubsection{Text Legibility}

This was assessed using black Tiresias [9] text on a white background with text decreasing in size from $84 \mathrm{pt}$ at the top to $2 \mathrm{pt}$ at the bottom.

On screen $A$, all text was readable down to and including the 3rd line from bottom (starting 'BK'). This line was Tiresias 6 pt text. On screen B, this same line (3rd from bottom) was still just about legible, but was harder to read.

On screens $C \& D$, all text was readable down to and including the 2nd line from bottom (starting ' $\mathrm{YN}$ '). This line was Tiresias $4 \mathrm{pt}$ text and was legible on both screens, but easier to read on $D$.

In summary then, text legibility was best on screen $D$ (15" transflective), followed by C (15" high-bright), A (12" high-bright with bonded A-R glass) and finally $B$ (12" transflective). However, it should be noted that these results reflect that the image was down-scaled to be displayed on 12" displays, i.e. text was larger on the 15 " displays.

\subsubsection{Colour Reproduction}

This was assessed using a cropped PDI colour chart [1] and a range of other exemplar customer ATM screens.

Screen A (12" high-bright with bonded A-R glass) was found to give the best colour reproduction followed by screen D (15" transflective), screen B (12" transflective) and finally screen C (15" highbright) being worst.

\subsubsection{Evaluation Under Moderate Lighting (22 k lux)} All displays were viewable at all head heights. Text legibility and colour reproduction were best for screen A (12" high-bright with bonded A-R glass), followed by screens B (12" transflective), C (15" high-bright) and $D$ (15" transflective). However, the performance of $B, C$ and $D$ seemed much closer under this lighting condition. 


\subsubsection{Evaluation Under Low Lighting (0 lux)}

All displays were viewable at all head heights. Text legibility and colour reproduction were acceptable for all screens with little to differentiate the displays. Brightness was evaluated in terms of comfort of use, with screen $D$ (15" transflective) being brightest under this lighting condition but not uncomfortable to use. Screens A (12" high-bright with bonded A-R glass), B (12" transflective), and C (15" high-bright) were approximately the same brightness under this lighting condition.

\section{EVALUATION 3: 5 DISPLAYS, 20 PARTICIPANTS}

\subsection{Method}

Five displays were evaluated under the same lighting conditions at the National Physical Laboratory (NPL). The displays were as follows:
A. 15" transflective concept (4 lamps)
B. 15" high-bright LED display
C. 12" high-bright bonded glass
D. 15 " transflective (2 lamps)
E. 15" standard bright with bonded glass

\subsubsection{Design}

A repeated measures design was used in this study. The principal factor was that of screen type with five levels corresponding to the different screens used. The dependent variables were subjective ratings of the display performance. Participants took part in all conditions with ordering of displays following a partial counterbalanced approach (Latin square).

\subsubsection{Participants}

20 people participated, 8 male and 12 female with all participants being NPL employees, and none having participated in the previous evaluation. The age of participants varied from 20 to $60+$ with the majority in the 30-39 and 50-59 age ranges, and height varied from $1524-1905 \mathrm{~mm}$ with a mean height of 1701 . All participants had normal or corrected to normal vision, and none reported any visual impairments that would interfere with their use of a display.

\subsubsection{Apparatus and Materials}

All displays were mounted at the same height and angle, which was $1400 \mathrm{~mm}$ from the floor and at $55^{\circ}$ from the horizontal (Figure 2). Displays were labeled by letter only (i.e. participants were not told which technology each display used). The height and angle chosen corresponded to the height and angle of the display in one of our ATMs.

The five displays were mounted on a movable platform which was moved to position the display under review centrally on the lighting arrangement (meaning that the same level of directed glare and indirect reflections were introduced on each display). Figure 2: Evaluation 3 Displays

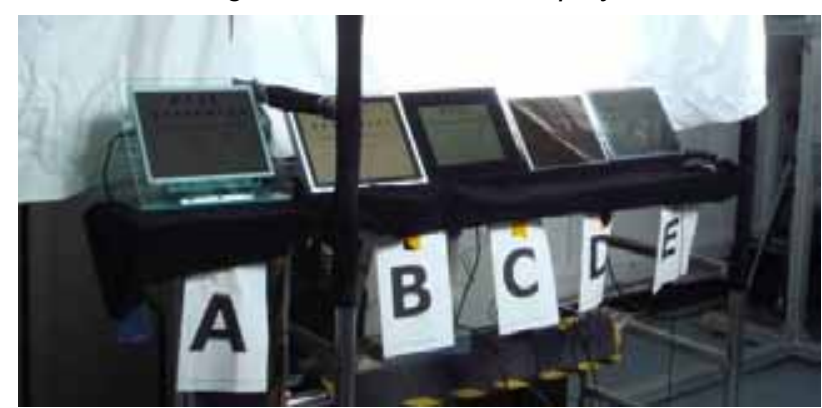

For this evaluation, all five screens had security glass placed in front with an anti-reflective coating on both the front and rear face of the glass. Panels $A, C, D \& E$ all had an anti-reflective coating. Screen $B$ had an anti-glare etch on the panel itself - this gave a matt finish that diffused reflections.

The displays were assessed under a single lighting state corresponding to bright, midday sunlight and was measured at 101,000 lux, where $76 \mathrm{~K}$ was direct light, $25 \mathrm{~K}$ was diffuse (Figure 3 ). In addition, the direct light was setup to replicate the situation with low sun shining over the participant's shoulder; one of the most challenging conditions to accommodate.

Figure 3: Evaluation 3 Lighting

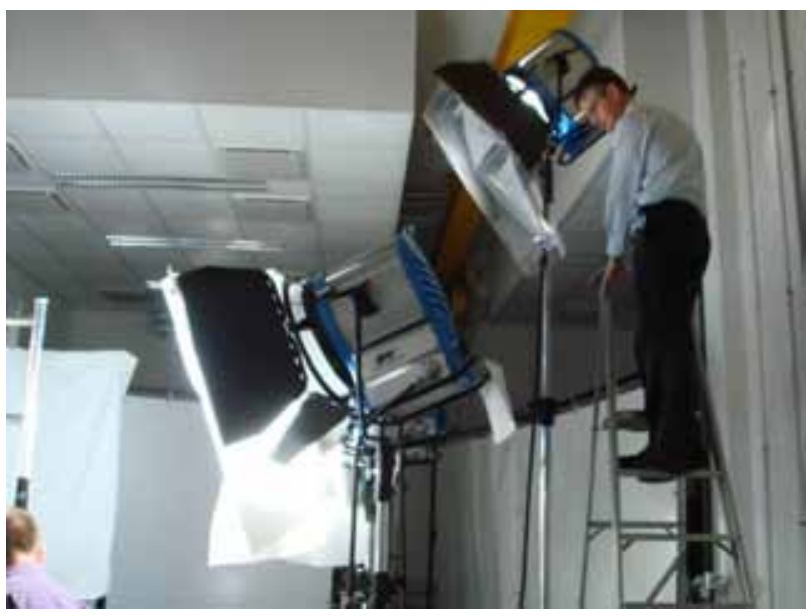

As in the first evaluation questions were asked before the evaluation regarding the age, gender, and visual acuity of a participant. After viewing images on each display a participant was then asked to rate them in terms of clarity of image, brightness, glare and comfort.

\subsubsection{Procedure}

Each participant completed the pre-experiment questionnaire before viewing standard test images and answering a few questions for each display. The test images were as in study 1 , with the exception of the snow scene that was omitted (Table 1).

Participants positioned themselves $300 \mathrm{~mm}$ from the bottom edge of the display in order to view the images, and were then asked to complete a post-experiment questionnaire eliciting attitudinal 
measures of perceived performance.

\subsection{Results}

Participants were asked to comment on screen clarity, brightness, comfort, glare/reflections and then give a comparison between the displays.

\subsubsection{Screen Clarity}

Participants were asked to rate this on a 5-point scale, where $1=$ very difficult to read, $5=$ very easy to read. Again, ranked scores were used to clearly show the difference between ratings. Ranked Score $=\left(a^{*} 1+b^{*} 2+c^{*} 3+d^{*} 4+e^{*} 5\right)$.

Ratings for this and other measures are summarized by means of the ranked scores in Table 4 for brevity.

\subsubsection{Screen Brightness}

This was also rated on a 5 point scale, but this time the midpoint ( 3 ) was the positive result; $1=$ too bright, $3=$ just right, $5=$ too dim. Ratings for this and other measures are summarized by means of the ranked scores in Table 4 for brevity. Ranked score $=$ $\left(a^{*} 1+b^{*} 3+c^{*} 5+d^{*} 3+e^{*} 1\right)$.

\subsubsection{Glare and Reflection}

This was also rated on a 5 point scale, where $1=$ big problem with glare/reflections, $5=$ no problem. Ratings for this and other measures are summarized by means of the ranked scores in Table 4 for brevity. Ranked Score $=\left(a^{*} 1+b^{*} 3+c^{*} 5+d^{*} 3+e^{*} 1\right)$.

No screens were rated very high for this, partly because the lighting condition was quite severe (with high intensity directed light analogous to a low sun facing the screen), and the displays all being assessed with security glass in front of them.

\subsubsection{Screen Comfort}

This was rated on a 5 point scale, where 1=very uncomfortable, $5=$ very comfortable. Ratings for this and other measures are summarized by means of the ranked scores in Table 4 for brevity.

Ranked Score $=\left(a^{*} 1+b^{\star} 2+c^{\star} 3+d^{*} 4+e^{\star} 5\right)$

\subsubsection{Comparison Between Screen Ratings Ratings} for each screen gave a clear indication of preference between the displays. When summarized by means of ranked scores for clarity, brightness and comfort, screens $D \& E$ were rated very poorly. Screens $A$, $B$ and $C$ were rated somewhat more highly, with $C$ being highest overall, and $A$ next highest.

However, it should be noted that the ratings were not highly positive. For instance, if we take a summation of the ranked scores, each display could score up to 320 (4 categories, 80 in each). Even A and $C$ are some way behind this maximum limit, and there is little to distinguish these two displays overall in terms of participant rating.
If we take the 4 ratings given for each display, we have $C$ as best, $A$ next, then $B$, then $D$ and finally $E$. When comparing the ratings for clarity, brightness, glare and comfort (combined into a single overall rating), there were significant differences (tested using Wilcoxon Signed-rank tests) between the following ratings

- $D$ and $A(T=-60.0, p<.01)$

- $\quad \mathrm{D}$ and $\mathrm{B}(\mathrm{T}=-44.5, \mathrm{p}<.05)$

- $\quad \mathrm{D}$ and $\mathrm{C}(\mathrm{T}=-71.0, \mathrm{p}<.005)$

- $\quad E$ and $A(T=-74.5, p<.001)$

- $\quad E$ and $B(T=-56.5, p<.05)$

- $\quad E$ and $C(T=-88.0, p<.0001)$

There were no significant difference between $B$ \&A, $C \& A, C \& B$, and $E \& D$. When comparing overall ratings for the displays (given by participants after using all displays and again tested using Wilcoxon Sign tests), there were significant differences between the following ratings

- $\quad D$ and $A(T=61.0, p<.05)$

- $D$ and $C(T=47.0, p=.056)$ - tending to significance $\cdot E$ and $A(T=64.0, p<.0005)$

- $E$ and $B(T=46.5, p<.05)$

- $\quad E$ and $C(T=67.0, p<.01)$

There were no significant differences between $B \& A$, $C \& A, C \& B$, and $E \& D$ as before. In addition, there was no significant difference between $D \& B$.

Table 4: Evaluation 3 Ranked Scores

\begin{tabular}{|l|rrrr|r|}
\hline & Clarity & Brightness & Glare & Comfort & Total \\
\hline A & 61 & 59 & 49 & 53 & 222 \\
B & 58 & 60 & 42 & 55 & 215 \\
C & 70 & 70 & 46 & 56 & 242 \\
D & 48 & 46 & 39 & 43 & 176 \\
E & 38 & 43 & 36 & 40 & 157 \\
\hline
\end{tabular}

4.2.6 Comparison Between Screen Opinions

Participants were also asked some general comparative questions at the end of the evaluation. A majority of participants (85\%) felt that there was a 
discernible difference between the displays.

Mean rankings for the displays ranked $C$ as first (2.3), A next (2.45), then B (2.8), D (3.55) and E was ranked last (3.8).

\subsection{Discussion}

From the ratings of clarity, brightness and comfort display $C$ (which had anti-reflective glass bonded directly onto the display panel) was preferred. However, this was judged to be due to the lack of air-gap in this setup rather than the fact that it was a high- bright backlit display. In fact, the other two 15 " high-bright displays evaluated ( $B$ and $E$ ) did not have this bonded glass, and were rated less highly by participants. Display A (15" transflective) was the next best after $A$ in terms of these ratings, followed by $B$ ( 15 " high bright LED), D (15" transflective, 2 lamps) and finally $\mathrm{E}$ (15" standard-bright).

This evaluation included a number of displays that were thought to be insufficient for the task (namely $D$ and $E$ ) due to the low level of illumination offered, and these results would support this initial hypothesis as both displays were rated poorly by all participants. From these results, if the bonded glass display is eliminated, the performance of the transflective displays was again at least as good as the highbright backlit display.

\section{EVALUATION 4: 5 DISPLAYS, EXPERT REVIEW}

\subsection{Results}

The first stage of this evaluation was conducted under bright light (101 k lux). Three experts (one usability specialist, one displays engineer, and one research scientist) evaluated the displays independently using the same criteria, results were then collated and trends identified.

\subsubsection{Viewability}

All displays were viewable at all likely user head heights; i.e. the display view angles accommodated everyone, with text contrast and colour reproduction being acceptable from all head heights. However, there were lots of glare issues which meant that one had to move around a lot to counteract the effects of glare and to successfully view portions of the display. It should be noted that display B had an anti-glare etch on the panel which diffused the light. This reduced the direct light reflected back, but in doing so tended to cause the surrounding area to haze (i.e. other displays had brighter sun spots, this one had a less bright but wider sun haze). This was good in that it reduced the intensity of reflected light, and as long as there was room to move around and get the sun 'haze' off the screen it was the best performing screen in this lighting condition.

\subsubsection{Text legibility}

This was assessed using black Tiresias text on a white background. A direct comparison of text legibility was not feasible as display $C$ was the only 12" panel. However, text legibility on this panel did seem good, although the 2nd line from the bottom could not be read due to the smaller image size.

\subsubsection{Colour reproduction}

This was assessed using a cropped PDI colour chart [1] as before.

\subsubsection{Summary}

Experts are identified as E1, E2 and E3 respectively. Results are summarized along with the participant rankings in Table 5.

Table 5: Summary of Expert Review Findings

\begin{tabular}{|l|l|l|l|l|l|}
\hline & $\begin{array}{r}\text { Best } \\
\text { (rank } \\
1)\end{array}$ & Rank 2 & Rank 3 & Rank 4 & $\begin{array}{r}\text { Worst } \\
\text { rank 5) }\end{array}$ \\
\hline $\begin{array}{l}\text { Participant } \\
\text { ranking } \\
\text { from } \\
\text { categories }\end{array}$ & $\mathrm{C}$ & $\mathrm{A}$ & $\mathrm{B}$ & $\mathrm{D}$ & $\mathrm{E}$ \\
\hline $\begin{array}{l}\text { Overall } \\
\text { participant } \\
\text { ranking }\end{array}$ & $\mathrm{C}$ & $\mathrm{A}$ & $\mathrm{B}$ & $\mathrm{D}$ & $\mathrm{E}$ \\
\hline E1 ranking & $\mathrm{C}$ & $\mathrm{A}$ & $\mathrm{B}$ & $\mathrm{E}$ & $\mathrm{D}$ \\
\hline E2 ranking & $\mathrm{B}$ & $\mathrm{A}$ & $\mathrm{C}$ & $\mathrm{D}$ & $\mathrm{E}$ \\
\hline E3 ranking & $\mathrm{C}$ & $\mathrm{A}$ & $\mathrm{B}$ & $\mathrm{D}$ & $\mathrm{E}$ \\
\hline
\end{tabular}

Displays D \& E were rated poorly by all participants. This finding was also supported by the expert evaluations conducted and so these displays should not be adopted an exterior installation.

There was some difference of opinion between expert findings on displays $A, B$ \& $C$.

In general, display C (12" high bright with bonded glass) was rated highest.

Displays A (15" transflective) and B (15" high bright) were rated next highest. A seemed to be slightly preferred overall, possibly due to the hazing effect exhibited by the anti-glare etch on display B. However, in reality there was little to differentiate these 2 displays. A brief comparison was made after the test between our existing 15" transflective panel and the proposed 15" transflective. Again, the illumination level offered by our current panel was superior to that of display $D$. 


\section{RESULTS}

\section{GENERAL DISCUSSION AND CONCLUSIONS}

\subsection{Summary}

The perceived performance and opinion of transflective displays was found to be at least as good as the conventional high-bright backlit displays in these adverse lighting conditions.

In addition, the performance of some displays with less illumination (standard bright backlit and transflective with only two lamps) was found to be unacceptable for use in bright sunlight.

The elimination of an air gap between the antireflective glass (containing the touch element) and the display panel by use of a 'bonded glass' approach was demonstrated to have a discernible advantage in concepts tested in the evaluations.

\subsection{Discussions From Evaluations 1 \& 2}

In general smaller 12" displays were preferred over 15 ".

The 15 " transflective display (D) was better than the 15 " high- bright $(C)$ in terms of participant ratings and results from the expert evaluation.

The 15" transflective display (D) also appeared to be slightly better than the 12" transflective (B) in terms of glare, text legibility, and colour reproduction when assessed in the expert evaluation. However, the preference for smaller screen size meant that participants still rated the 12" transflective (B) slightly higher than the 15 " transflective (D) in terms of participant ratings for clarity, brightness and comfort. These results therefore suggest that the performance of the 15 " transflective display is no worse that that of a comparable 15" high-bright and may be better in some lighting conditions (sunlight) as the antireflective coating used on transflective displays gives much better reduction of reflections than the antiglare etch used on high-bright displays.

However, it is not clear from these results whether a high-bright display with an anti-reflective coating would be superior to a transflective display; certainly if this a-r coating is bonded directly onto the glass the display would be better (as demonstrated by screen A in the study).

The display with bonded A-R glass $(A)$ was rated significantly higher than those displays with separate glass placed on the front of the display (i.e. A much better than $B, C, D$ ) particularly in participant ratings for image clarity. Future use of this type of display would appear to give significant benefits in terms of image quality to the user.

\subsection{Discussions From Evaluations 3 \& 4}

When comparing the ratings for clarity, brightness, glare and comfort and overall ratings for displays there were some significant differences as previously reported.

In general, these results indicate that displays $D$ \& $E$ were significantly worse than $A, B$ \& $C$ both in terms of overall subjective ratings, and in terms of perceived clarity, brightness and ability to cope with glare. Illumination levels provided by these displays are insufficient for use in a bright sunlit environment such as can be experienced in an exterior installation.

\subsection{Study limitations \& Lessons Learnt}

There were a number of limitations of this study. Apparatus differences. Firstly, the exact setup between the two sets of displays varies somewhat. The lighting setup, although similar, did vary slightly, as did the height that displays were evaluated. This was due to the time difference between the two studies, and slight differences in experimental equipment.

Sample size. Another limitation was the relatively small sample sizes (16 and 20 ) used. This meant that the effect of factors such as age, gender, or height of the participants (which would subdivide the sample resulting in very small groups) could not adequately be assessed as little confidence can be placed in the statistical validity of such analysis.

Backlight differences. In the first study there seemed to be a difference in performance between the 2 transflective screens - when the effects of glare were excluded (i.e. under diffused light), colour reproduction and image clarity was better on the 15 "transflective than on 12". This was due to the 15 " having a brighter backlight (600 lux rather than a specified 400 on 12"). This may well account for some of the difference in ratings between these screens.

In addition to these areas of limitation, there were also some lessons to be learnt from these studies.

Screen sizes. Both 12 and 15 " variants of displays were evaluated. It was difficult to compare text legibility between these panels using the setup used in these two tests (where the same image was fed to all displays, and the display scaled to fit). Future evaluations should consider techniques for presenting different sizes of images for the different panel sizes.

Illumination conditions. This evaluation was an attempt to test the worst case; namely direct glare onto the screen. A more representative test would have used multiple lighting conditions to best balance overall performance.

Use current display as control. For future tests it is strongly recommended that our current display be 
used as the control against which direct comparisons can be made.

Limit study scope. It is also recommended that the number of displays be limited to four. If more displays are to be tested, then splitting the numbers and running multiple tests may be more useful. (Some participants were complaining of visual fatigue when evaluating the five displays under a high-glare situation.)

\subsection{Conclusions}

There are a number of conclusions that can be drawn from these studies. The first, and most significant, is that we have found transflective displays to be as good as high bright displays, and as they offer both cost and environmental advantages they should be preferred over high-bright alternatives.

The use of displays where the glass is bonded directly onto the display panel (thus eliminating the air gap) appears to be very promising in terms of customer acceptance as demonstrated in the four evaluations, but the technology requires further investigation into other factors including total cost of ownership, display lifetime and the resistance of the solution to damage (vandal resistance).

In addition, the second study clearly demonstrates that there is a minimum level of illumination that is appropriate for a display to be acceptable for use in an exterior environment.

Anti-glare etches and matt finishes, although successfully reducing the direct reflections of glare and reflections do not appear to be suitable for exterior use as they add the additional complication of a bright haze obscuring the screen image. It is therefore recommended that anti-reflective coatings are more appropriate in an exterior environment.

Finally, it is interesting to note that although the trend in display manufacturers is to larger format panels, smaller displays may be helpful in dealing with glare and reflections.

Although this work has focused on the use of displays in self- service terminals, it is also of relevant to other application areas that make use of displays in exterior environments such as mobile telephones (especially smart phones), portable computing (such as laptops), dynamic information displays and digital signage.

\section{ACKNOWLEDGMENTS}

We are grateful to the National Physical Laboratory (NPL) for their support in completing this study, and to NCR Corporation for commissioning this activity.

\section{REFERENCES}

[1] PDI freeware test image. From http://www. northlightimages. co.uk/article_pages/test_images. html.

[2] K. T. Burnette. WL-TR-91-7020 an evaluation of aircraft CRT and dot-matrix display legibility requirements. Technical report, Wright Laboratory, Air force material command, June 1994.

[3] H.-H. Chung and S. Lu. Contrast-ratio analysis of sunlight- readable color LCDs for outdoor applications. Journal of the Society for Information Display, 11(1):237-242, 2003.

[4] E. G. Conlon, W. J. Lovegrove, E. Chekaluk, and P. E. Pattison. Measuring visual discomfort. Visual Cognition, 6(6):637-663, December 1999.

[5] P. N. Day, C. Rohan, L. Coventry, G. I. Johnson, and C. Riley. Reach modelling for driveup self-service. In Proceedings of the Institute for Ergonomics and Human Factors Annual Conference 2010, 2010. In press.

[6] D. G. Hopper. Cockpit display requirements and specifications. In Proceedings SPIE (The International Society for Optical Engineering), volume 1988, 1993.

[7] R. Sharpe, C. M. Cartwright, W. A. Gillespie, K. Vassie, and W. C. Christopher. Sunlight readability of displays: a numerical scale. In A. Springsteen and M. Pointer, editors, Fourth Oxford Conference on Spectroscopy, volume 4826, pages 176-180. SPIE, 2003.

[8] B. Shepherd. Electroluminescent instrumentation. Displays, 2(7):337 - 340, 1981.

[9] J. H. Silver, J. M. Gill, and J. S. W. Wolffson. Text display preferences on self-service terminals by visually disabled people. Optometry Today, 35(2):24-27, 1995.

[10] K. T. Smith and L. Coventry. Designing for a world population. In Contemporary Ergonomics 2002, pages 340-345, London, 2002. Taylor and Francis.

[11] C. K. Vassie and W. C. Christopher. Just acceptable and desirable luminance levels for fast jet cockpit displays. In D. G. Hopper, editor, Cockpit Displays VII: Displays for Defense Applications, volume 4022, pages 116-125. SPIE, 2000.

[12] A. Wilkins, I. Nimmo-Smith, A. Tait, C. Mcmanus, S. D. Sala, A. Tilley, K. Arnold, M. Barrie, and S. Scott. A neurological basis for visual discomfort. Brain, 107(4):989-1017, December 1984.

[13] X. Zhu, Z. Ge, T. X. Wu, and S.-T. Wu. Transflective liquid crystal displays. Journal of Display Technology, 1(1):15- 29, September 2005. 